\title{
A DENSE DISPARITY ESTIMATION METHOD USING COLOR SEGMENTATION AND ENERGY MINIMIZATION
}

\author{
Han Shin Lim and HyunWook Park \\ Dept. of Electrical Engineering, Korea Advanced Institute of Science and Technology \\ 373-1 Guseong-dong, Yuseong-gu, Daejeon 305-701, Korea \\ hwpark@athena.kaist.ac.kr
}

\begin{abstract}
This paper presents a new dense disparity estimation method using color segmentation and energy minimization. Segmentation-based methods have an advantage of clear representation of disparities on discontinuous region. This paper remodels the energy minimization method to fit with segmentation-based method.

In the proposed method, the initial disparity map is obtained by variable block matching of the segmented plane and RANdom SAmple Consensus (RANSAC) fitting. Then energy minimization is performed on the small segmented planes and the overall segmented planes, respectively to refine the disparity map more accurately. At the end of the method, erroneous stripes that are not corrected by the segmentation-based approach are processed by a pixelbased method.

Experimental results show that the error rate of the proposed method is comparable to other state-of-the-art matching methods on various stereo image pairs.
\end{abstract}

Index Terms-Stereo vision, Image matching, Machine vision

\section{INTRODUCTION}

Stereo correspondence is to determine the locations in each camera image that are the projection of the same 3D scene point. And it has become an important problem in image processing and computer vision communities. More accurate matching process and disparity map guarantee more reliable depth estimation and $3 \mathrm{D}$ reconstruction. In addition, the estimated disparity map can be used to compress the multi-view images [4].

Stereo disparities are determined in a number of ways and by exploiting a number of constraints. Generally, global correspondence methods exploit global constraints in order to reduce sensitivity to local regions in the image that fail to match, due to occlusion, uniform texture, etc. The use of these constraints makes the computational complexity significantly greater than that of local matching [3].
In this paper, we present a new disparity estimation method that is using mean shift-based color segmentation [5] and global energy minimization methods. We remodeled the energy minimization algorithm that can be applied to the segmentation-based method.

\section{PREVIOUS WORKS}

\subsection{Incremental Warping [1]}

In the proposed algorithm, the second view image is first predicted from the reference view image using the depth information. Then, find the depth map that maximizes the similarity between the predicted image and the actual image. The main advantage of this approach is that the occlusion problem of the segmentation-based algorithm can be considered.

Step 1. Base warp: In the warped image, save the information (segment ID and depth) of the two top-most layers (warped pixels) for each position $i$. Then, compute the matching score $c=\exp \left\{-\left(I-I^{\prime}\right)^{2} / 2 \sigma^{2}\right\}$, where $I$ and $I^{\prime}$ is the image intensities of a pixel in the second view and the base warp and $\sigma$ is a constant.

Step 2. Segment removal: Remove the pixels in a segment of the warped image. If some pixels are affected by the removal, update the recorded information (segment ID, depth and matching cost of each layer).

Step 3. Hypothesis testing: For each depth hypothesis of a segment i, warp the segment using the corresponding hypothesis and compute the global matching score. The test is performed on all segments.

Step 4. Update depth: After the hypothesis test, update the depth values. Step1 to Step 4 are iteratively performed until either the number of iterations reaches a maximum value or the global matching score converges.

\subsection{Cost Relaxation Energy Minimization [2]}

In the algorithm, the energy minimization process for disparity estimation is modeled by a modified differential 
equation system of order variables. Overall algorithm is described as follows.

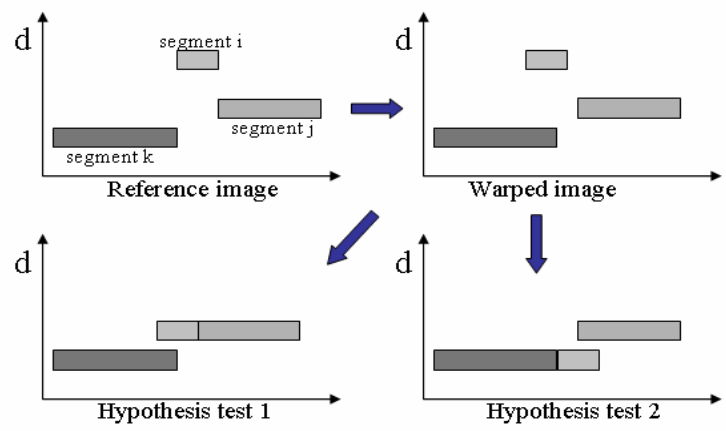

Fig.1. Incremental warping process.

Step 1. Similarity measure: normalized cross-correlation vector windowed and weighted by a Gaussian or uniform function $f$ is defined as

$$
\xi=\left(\xi_{\left(1, d_{\min }\right)}, \ldots, \xi_{\left(n, d_{\min }\right)}, \xi_{\left(1, d_{\min }+1\right)}, \ldots, \xi_{\left(n, d_{\max }\right)}\right)^{T}
$$

Step 2. Global optimization via cost-relaxation: The cost function is the sum of the data term and smoothness term:

$$
P(\xi)=P_{1}(\xi)+P_{2}(\xi)
$$

where the data term and smoothness term are formulated as

$$
\begin{gathered}
P_{1}(\xi)=c_{1} \sum_{d=d_{\min }}^{d_{\max }} \sum_{i=1}^{n}\left(\xi_{(i, d)}-\xi_{(i, d)_{0}}\right)^{2} \\
P_{2}(\xi)=c_{2} \sum_{d=d_{\min }}^{d_{\max }} \sum_{i=1}^{n} \sum_{j \in U_{i}} w_{i j}\left(\xi_{(i, d)}-\xi_{(i, d)_{0}}\right)^{2}
\end{gathered}
$$

$c_{1}$ and $c_{2}$ are the positive constant weights, and $U_{i}$ represents the local support area of $i$, The minimum of $P(\xi)$ has to satisfy

$$
\frac{\partial P}{\partial \xi_{(i, d)}}=0
$$

The minimum can be computed numerically with the gradient descent method.

Step 3. Disparity estimation: The disparity value that has maximum parameter belonging to $k$ is determined to estimate disparity $d(k)$.

$$
d(k)=\left.j\right|_{\xi(k, j)=\max (\xi(k, i))}
$$

with $i \in\left\{d_{\min }, \ldots, d_{\max }\right\}$.

\section{PROPOSED ALGORITHM}

This section describes the proposed dense disparity estimation algorithm. Figure 2 is the overall flowchart of the proposed method.

At first, the reference image is oversegmented by using the mean-shift color segmentation [2]. And segmentation ID is assigned to each segmented plane.

\subsection{Variable Block Matching}

After the color segmentation, variable block matching using information of the segmented planes is applied to initialize the disparity map. Matching cost of the variable block matching is the Normalized Cross Correlation (NCC). And left-right consistency checking is applied to verify the matching reliability. Then, matching cost $c(x, y, d)$ is rescaled within a range between 0 and 1 .

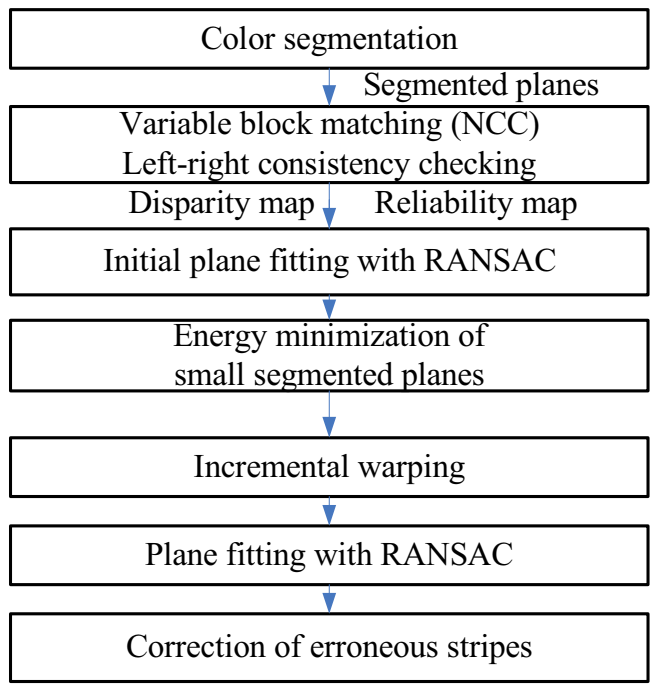

Fig. 2. Flowchart of the proposed method.

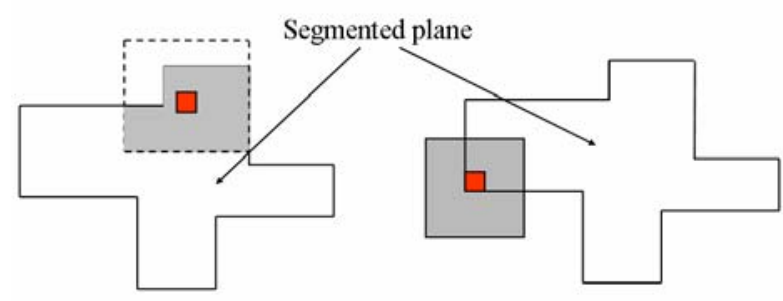

(a)

(b)

Fig. 3. Variable block matching. (a) When a pixel is not on the boundary of a segmented plane, (b) When a pixel is on the boundary.

If a pixel is located in a segmented plane, pixels that are outside the segmented plane are removed from the block and block matching is processed. And block matching is processed using the block. But if a pixel is on the boundary, fixed block matching is applied.

\subsection{Plane fitting with RANSAC}

RANSAC uses a small initial data set as feasible and enlarges this set with consistent data if possible [7]. If the model is a plane, then the minimum number of data to fit a plane is three. So RANSAC procedure to fit a plane is given as follows.

1. Repeat for $\mathrm{N}$ samples.

(a) Select randomly three samples a, b, c. 
(b) Compute the plane equation.

(c) Count the number of inliers.

(d) Choose a data set that maximizes the number of inliers.

2. Fit a plane from all samples classified into inliers.

3. Calculate $\xi_{(i, d)_{0}}$ (the initial probability that the $i$ th segmented plane has disparity $d)$.

$$
\xi_{(i, d)_{0}}=\frac{\sum_{(x, y) \in i} c(x, y, d)}{\sum_{d=d_{\min }}^{d_{\max }} \sum_{(x, y) \in i} c(x, y, d)}
$$

where $i$ is the set of inliers.

\subsection{Energy Minimization of The Segmented Planes}

To estimate and refine disparities of the small segmented planes, we used energy minimization method based on cost relaxation method. Definition of the small segmented plane is a segmented plane that has a number of reliable pixels less then $N_{\text {threshold }}$. If the number of reliable points is less then three, then a plane cannot be fitted and $\xi_{(i, d)_{0}}$ cannot be calculated. In this case, $\xi_{(i, d)_{0}}$ is assigned as

$$
\xi_{(i, d)_{0}}=\frac{1}{d_{\max }-d_{\min }}
$$

If $3 \leq n_{\text {reliable }}<N_{\text {threshold }}\left(n_{\text {reliable }}\right.$ : number of reliable pixels), then the segmented plane is fitted from all $n_{\text {reliable }}$ points using least squares method. In this case, if the least squares method can be applied, $\xi_{(i, d)_{0}}$ is assigned as (6). Otherwise $\xi_{(i, d)_{0}}$ has the uniform distribution as (7).

Each part of the energy formula is modeled as follows

$$
P_{1}(\xi)=c_{1} \sum_{d=d_{\min }}^{d_{\max }} \sum_{i=1}^{m}\left(\xi_{(i, d)}-\xi_{(i, d)_{0}}\right)^{2}
$$

where $c_{1}=n_{\text {reliable }}, m$ is the number of segmented planes

$$
P_{2}(\xi)=c_{2} \sum_{d=d_{\min }}^{d_{\max }} \sum_{i=1}^{m} \sum_{j}^{m} w_{i j}\left(\xi_{(i, d)}-\xi_{(j, d)}\right)^{2}
$$

where $w_{i j}$ is the number of boundary lines of $j$ th large plane that surround the $i$ th small plane.

The more $i$ th small segmented plane has $n_{\text {reliable }}$, the stronger the data term is. And the number of boundary pixels of $i$ th small segmented plane is related to the strength of the smoothness term.

To obtain a minimum energy, we use gradient descent method. Then, each disparity is assigned to each small segmented plane.

After the energy minimization of the small segmented planes, the incremental warping process is performed for all segmented planes. In the process, smoothness term and color similarity term is not used.

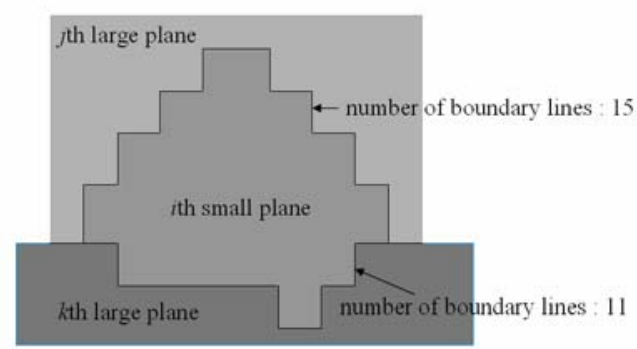

Fig.4. Boundary lines of each neighborhood segmented plane.

\subsection{Reduction of Erroneous Stripes}

After the energy minimization, a pixel-based process to reduce the erroneous stripes is applied. The procedure is given as follows.

1. For all pixels, check if the left and right pixels or upper and lower pixels of a pixel have all different segmentation ID (the distance of each pixel is one or two or three).

2. If the three pixels have all different segmentation IDs, check each pixel's estimated disparity.

3 . If at least one of these pixels has different disparity, reestimate the disparity of center pixel using variable block matching. Search range is only the disparity values of the center pixel and other two pixels.

4. If re-estimated disparity value is different from its former value, check the consistency of the re-estimated disparity value using left-right consistency checking.

5. If it passes the checking process, determine the disparity of the center pixel as the re-estimated disparity value. And reassign the segmentation ID of the center pixel.

6. Repeat 1 5 until all disparities are not changed.

\section{EXPERIMENTAL RESULTS}

Experimental results on test stereo image pairs with ground truth are shown in this section. Intel Pentium-IV $2.6 \mathrm{GHz}$ was used and our algorithm was implemented by Visual $\mathrm{C}++6.0$. The epipolar lines of all stereo image pairs are on the scanline. In the variable matching process, we set the block size to $7 * 7$.

The error rate was computed as

$$
B=\frac{1}{N} \sum_{(x, y)}\left(\left|d_{g t}(x, y)-d_{e s t}(x, y)\right|>1\right)
$$

where $N$ is the total number of pixels that are not lying in occluded regions. This measurement method is same as [6].

We compared the error rates of our proposed method with the other state-of-the-art global matching methods.

Table.1 shows the accuracy of our algorithm is comparable to other state-of-the-art disparity estimation methods. And the processing time is similar to those of the compared method. 
(a)
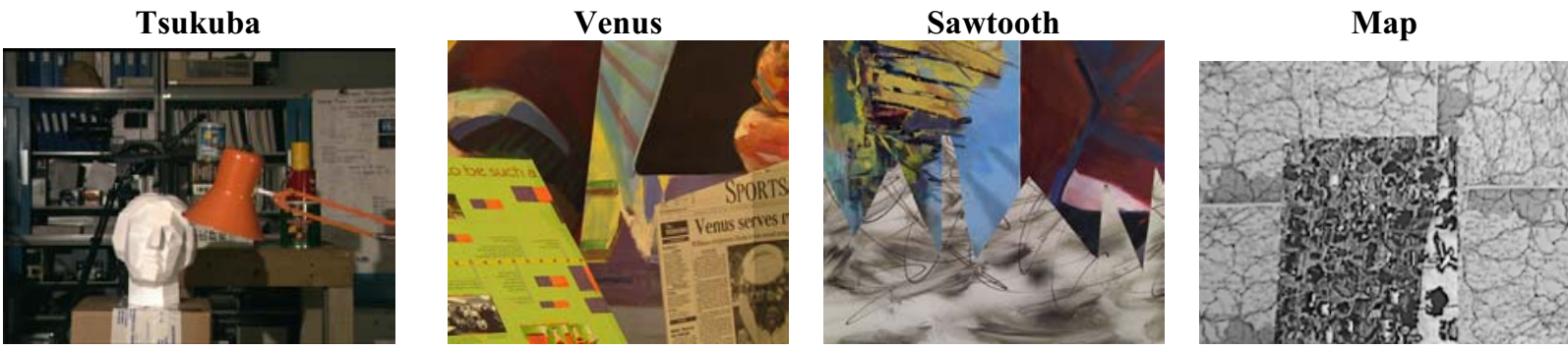

(b)
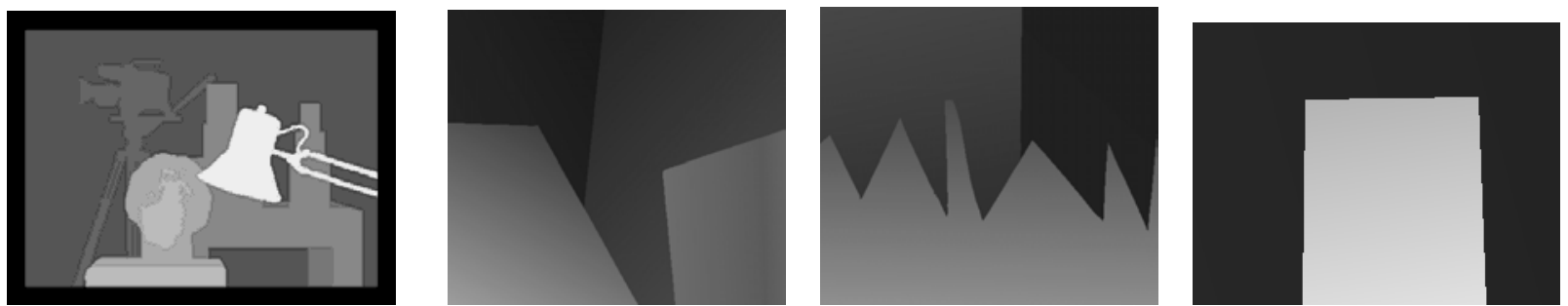

(c)
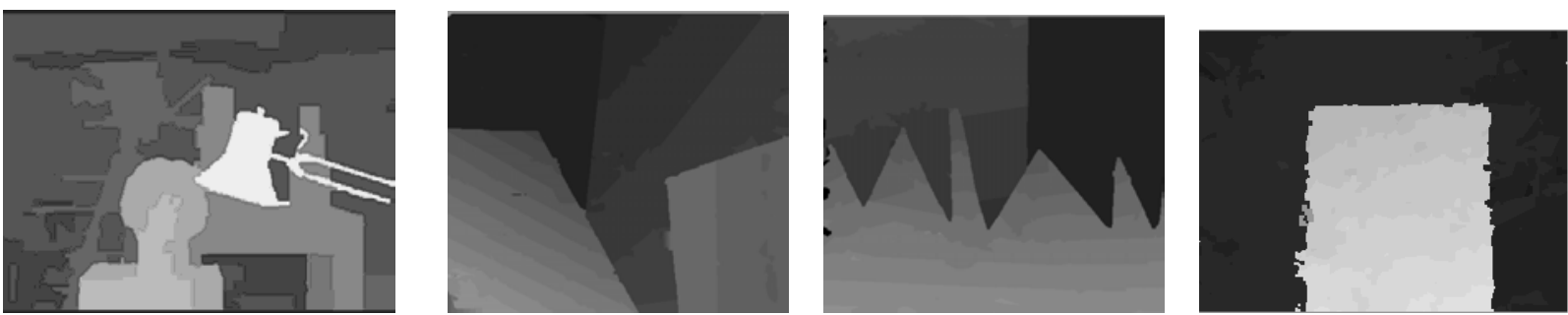

Fig.5. Disparity estimation results on test image pairs (a) left image, (b) ground truth,

(c) obtained disparity map from the proposed method.

Table.1. Comparisons of error rate with the state-of-theart matching methods (\%).

\begin{tabular}{|c|c|c|c|c|}
\hline & Tsukuba & Venus & Sawtooth & Map \\
\hline Sym.BP+occl. & 0.97 & 0.16 & 0.19 & 0.16 \\
\hline Patch-based & 0.88 & 0.09 & 0.25 & 0.30 \\
\hline Proposed & $\mathbf{1 . 0 3}$ & $\mathbf{0 . 3 3}$ & $\mathbf{0 . 3 0}$ & $\mathbf{0 . 8 4}$ \\
\hline $\begin{array}{c}\text { Segm.-based } \\
\text { GC }\end{array}$ & 1.23 & 0.08 & 0.30 & 1.49 \\
\hline Graph+segm. & 1.39 & 0.11 & 0.25 & 2.35 \\
\hline
\end{tabular}

From http://cat.middlebury.edu/stereo/

\section{CONCLUSIONS}

We proposed a new disparity estimation method based on color segmentation and energy minimization. The energy minimization method was remodeled to fit with segmentation-based method. And the pixel-based correction process was applied to reduce erroneous disparities that are not corrected by the segmentation-based method.

Our method was compared with state-of-the-art matching methods. Experimental results showed error rate of our algorithm is comparable to those of other compared methods. And processing time was also reasonable.

\section{ACKNOWLEDGEMENT}

This research was supported in part by University IT Research Center Project.

\section{REFERENCES}

[1] H. Tao, H. S. Sawhney and R. Kumar, "A Global Matching Framework for Stereo Computation," Proc. Int'l Conf. Computer Vision, 2001.

[2] R. Brockers, M. Hund and B. Mertsching, "Stereo Matching with Occlusion Detection Using Cost Relaxation," IEEE Conf. on Image Processing, 2005.

[3] M.Z. Brown, D. Burschka, and G.D. Hager, "Advances in Computational Stereo," IEEE Trans. on Pattern Analysis and Machine Intelligence, Vol. 25, No. 8, pp. 993-1008, 2003.

[4] J.H. Park and H.W. Park, "A mesh-based disparity representation method for view interpolation and stereo image compression," accepted to IEEE Trans. on Image Processing, 2005.

[5] D. Comaniciu and P. Meer, "Robust Analysis of Feature Spaces: Color Image Segmentation," IEEE Conf. on Computer Vision and Pattern Recognition, 1997.

[6] D. Scharstein and R. Szeliski, "A Taxonomy and Evaluation of Dense Two-Frame Stereo Correspondence Algorithms," Int'l J.Computer Vision, vol. 47, no. 1, pp. 7-42, 2002.

[7] R. Hartley and A. Zisserman, Multiple View Geometry in Computer Vision. Second Edition, Cambridge: UK, Cambridge Univ. Press, 2003.

[8] http://cat.middlebury.edu/stereo/. 